Chirurg 2021 · 92:479

https://doi.org/10.1007/s00104-021-01416-7

Angenommen: 26. März 2021

Online publiziert: 20. April 2021

(c) Springer Medizin Verlag GmbH, ein Teil von Springer Nature 2021

\section{Originalpublikation}

Hasselgren K, Røsok BI, Larsen PN et al (2021) Survival compared with TSH in patients affected of CRLM: survival analysis from the randomized controlled trial LIGRO. Ann Surg 273(3):442-448. https://doi.org/10.1097/SLA. 0000000000003701

Hintergrund. Patienten mit ausgeprägter Lebermetastasierung haben eine schlechte Prognose und sind schwierig zu behandeln. Das meist zu erwartende zu geringe postoperative Lebervolumen limitiert die chirurgischen Interventionsmöglichkeiten.

Methodik. Im Rahmen der multizentrischen prospektiven LIGRO-Studie wurden im Zeitraum zwischen August 2014 und Juni 2016 insgesamt 100 Patienten mit kolorektalen Lebermetastasen und verbleibendem Lebervolumen von $<30 \%$ eingeschlossen. Voraussetzung für den Einschluss war eine Tumorkontrolle unter „neoadjuvanter“ Chemotherapie. Es erfolgte die randomisierte Zuteilung in einem 1:1-Verhältnis in die Resektion mittels ALPPS („associating liver partition and portal vein ligation in staged hepatectomy“, „In-situ-Split“) oder TSH („two-stage hepatectomy“, zweizeitige Leberesektion). In der Gruppe der TSH bestand die Möglichkeit, bei unzureichender Leberhypertrophie ALPPS im Sinne einer Rescue-Therapie durchzuführen. Die Nachsorge erfolgte mittels Schnittbildgebung 4 Wochen postoperativ, bei bestehender Indikation erfolgte ab diesem Zeitpunkt eine Chemotherapie. Weitere Bildgebungen wurden nach 4, 8, 12, 18 und 24 Monaten durchgeführt.

Michael Ardelt · Friedrich Köpp · Utz Settmacher

Klinik für Allgemein-, Viszeral- und Gefäßchirurgie, Jena, Deutschland

\title{
Kolorektale Lebermetastasen: eine randomisierte prospektive Analyse
}

Ergebnisse. In der ALPPS-Gruppe wurden 2 Patienten und in der TSH-Gruppe 1 Patient ausgeschlossen. Die Gründe waren jeweils Nichterfüllung der Einschlusskriterien. Die Auswertung erfolgte gemäß dem Intention-to-treatProtokoll. Die 90-Tage-Mortalität nach der zweiten Operation unterschied sich nicht signifikant. In der ALPPS-Gruppe konnten $92 \%$ der Patienten erfolgreich reseziert werden, in der TSH-Gruppe $80 \%(p=0,091)$. In der ersten postoperativen Bildgebung zeigten sich signifikant mehr Patienten der ALPPS-Gruppe mit tumorfreier Leber ( $77 \%$ vs. $57 \%$, $p=0,028)$. Die mediane Überlebenszeit war 46 Monate für ALPPS und $26 \mathrm{Mo-}$ nate für TSH $(p=0,028)$. Die mediane Überlebenszeit ab Diagnosezeitpunkt der hepatischen Metastasierung war für ALPPS 56 Monate und für TSH 38 Monate $(p=0,020)$. Im Vergleich mit erfolgreich resezierten Patienten zeigte sich ein tendenziell längeres Überleben der ALPPS-Patienten, jedoch kein signifikanter Unterschied. Patienten, bei denen ein „Rescue-ALPPS“ durchgeführt wurde, zeigten eine kürzere mediane Überlebenszeit als bei erfolgreicher Resektion bezüglich der initialen Randomisierung. Bezüglich der nichtresezierten Patienten beider Gruppen zeigte sich kein signifikanter Unterschied der Überlebenszeit bei schlechter Prognose. Die Regressionsanalyse zeigte, dass die Randomisierung zu ALPPS, die erfolgreiche Resektion der Lebermetastasen, der ASA-Status (American Society of Anesthesiologists), die maximale Metastasengröße sowie das Auftreten schwerer postoperativer Komplikationen mit der Prognose korrelierten.

\section{Kommentar}

Insgesamt hat das untersuchte Patientenkollektiv eine schlechte Prognose und eine hohe postoperative Morbidität und Mortalität. ALLPS hat im Rahmen dieser Studie eine signifikant längere Überlebenszeit im Vergleich zu TSH gezeigt. Die Ursache dafür ist wahrscheinlich die höhere Resektionsrate bei ALPPS. In den Subgruppen der erfolgreichen Resektion bestand kein signifikanter Unterschied der Überlebenszeit. Die Ergebnisse sind durch das multizentrische Design der Studie sowie die prospektive Auswertung qualitativ hochwertig. Schwächen der Studie sind die Patientenzahl sowie unterschiedliche Chemotherapien. Eine weitere methodische Schwäche ist sicherlich die Möglichkeit des RescueALPPS, da hiermit die Vergleichbarkeit der Gruppen schwieriger wird. Es ist festzuhalten, dass von den festgestellten Prognosefaktoren nur die Allokation zu ALPPS zum Diagnosezeitpunkt beeinflusst werden kann.

\section{Korrespondenzadresse}

PD Dr. Dr. Michael Ardelt

Klinik für Allgemein-, Viszeral- und Gefäßchirurgie

Am Klinikum 1, 07747 Jena, Deutschland

Michael.Ardelt@med.uni-jena.de

Interessenkonflikt. M. Ardelt, F. Köpp und U. Settma cher geben an, dass kein Interessenkonflikt besteht. 